\title{
Probing bio-molecules across polyelectrolyte multilayers
}

\begin{abstract}
Highly tuneable architecture of PEMs with nanometres precision is the most promising aspect to utilize them for variety of applications. The bulk loading properties of polyelectrolyte multilayer thin films (PEM) motivate researchers to utilize them as a carrier for biomedical applications. For designing an efficient carrier polymeric assembly for targeted and controlled drug delivery, it is very important to understand the loading and release kinetics of bio-molecules across PEM matrix. Therefore, a better and complete understanding of the molecular diffusion is highly recommended to achieve success in these areas. This mini review provides an overview of the current efforts in understanding the biomolecular transport across the polymer thin films. Different strategies involving confocalbased approaches along with recent innovative techniques have been briefly explained here to probe biomolecules in PEM. Pros and cons of each technique with future perspectives are discussed to render the most suitable technique for understanding the permeability of desired polymer matrix
\end{abstract}

Keywords: polyelectrolyte multilayer films, bioactive,fluorescence, microscopes
Volume 5 Issue 6 - 2017

\author{
Suman Pahal,' Ruchi Gakhar, ${ }^{2}$ Ashok M \\ Raichur, ${ }^{3,4}$ Manoj MVarma \\ 'Centre for Nano Science and Engineering, Indian Institute of \\ Science, India \\ ${ }^{2}$ Department of Engineering Physics, University of Wisconsin, \\ USA \\ ${ }^{3}$ Department of Materials Engineering, Indian Institute of \\ Science, India \\ ${ }^{4}$ Nanotechnology and Water Sustainability Unit, University of \\ South Africa, South Africa
}

Correspondence: Manoj MVarma, Centre for Nano Science and Engineering, Indian Institute of Science, Bangalore-5600 I2, India, Email mvarma@cense.iisc.ernet.in

Received: June 14, 2017| Published: August 01, 2017
Abbreviations: PEM, polyelectrolyte multilayer; FRAP, fluorescence recovery after photobleaching

\section{Introduction}

Polyelectrolyte multilayer films (PEM) are the most potential candidate for drug delivery applications as they offer stimuli sensitive and well-tuned bulk architecture with nanometres precision for loading of drug or bioactive species. ${ }^{1-4}$ For an efficient loading and targeted release of bio-active molecules, it is very important to understand the availability of encapsulated drug or bio-molecules within the polymer matrix. ${ }^{5,6}$ This local availability of the incorporated molecules is dependent on the transport properties of the particular PEM stack and is usually measured by the displacement of the adsorbed molecule in a given time scale with respect to its initial position or from the interface of loading. For tracking bio-molecules or probing biomolecular location across PEM matrix, it is important to apply the best technique without compromising the PEM's structural integrity. So far, researchers have explored a variety of techniques to estimate bio-molecular transport with varying degree of success. The main aim of this mini review is to provide an overview of the present research focused on understanding the bio-molecular transport in polymer thin films. Such an effort will lead to the design of better host materials for drug loading and various other bioactive species for the advancement of multi-component, targeted and controlled drug delivery applications.

\section{Confocal -based techniques}

Optical techniques such as confocal microscopy are based on fluorescence from the diffusing species. The salient features of confocal microscopes over the conventional fluorescence microscopes, such as better resolution and improved contrast, render it suitable to study molecular dynamics in biological samples. Moreover, confocal microscopy also provides three dimensional transport information in case of thicker samples by offering the optical sectioning and image reconstruction options. In order to acquire accurate, time-resolved information of molecular transport and to understand details of molecular interactions, a number of technical modifications have been implemented in the conventional confocal systems. Fluorescence Recovery after Photobleaching (FRAP), Forster Resonance Energy Transfer (FRET) and Fluorescence Correlation Spectroscopy (FCS) are the examples of such modified versions of conventional confocal microscopes. Table 1 discusses the detailed overview of the techniques mentioned in this review.

Fluorescence recovery after photo bleaching is the most versatile optical technique confocal microscopy for studying molecular transport within the biological cells. FRAP relies on the photoinduced bleaching of a region of interest (ROI) by utilizing a high intensity laser and monitoring the change in fluorescence intensity with time scale in order to track the molecular diffusion from the area surrounding bleached ROI. ${ }^{7}$ The recovery or the change in the fluorescence intensity of the ROI contains the information about the diffusion of molecules, which are labelled with fluorophores and diffusion coefficients can be extracted by fitting this data to various mathematical models. Existing literature contains a number of examples where FRAP has been employed to study bio-molecular transport in PEMs. ${ }^{8-11}$ In fact, FRAP is the most used technique for studying the lateral molecular transport across PEM. ${ }^{9,10}$ Very recently, FRAP has been utilized to study the interaction and loading behaviour of Lysozyme protein and Human Serum Albumin (HSA) across the polymeric stack of Poly(L-lysine) (PLL) and hyaluronic acid (HA) ${ }^{8,9}$ Due to the exponential layer growth in this system, this PEM stack is thick enough to be explored for excellent loading (Table 1).

FRAP has proved to be a versatile tool here, as it helps in studying the protein dynamics across PEM with different PEM compositions and varying physico-chemical surroundings, such as the terminating polymer layer, the deposition concentration, temperature, ionic strength and $\mathrm{pH}$ of the PEM constituents. In order to better understand the bio-interactions, FRAP is also used to detect differences in the diffusion behaviour of different proteins with varying molecular sizes within the same PEM stack. ${ }^{11}$ A major limitation of FRAP is that it can only be employed to explore molecular transport in PEM films with thickness more than a micron because of limited Z-resolution of confocal microscopy. 
Table I Provides an overview of the recent research activities which are focused on probing the bio-molecular transport across PEM

\begin{tabular}{|c|c|c|c|}
\hline Technique & Bio-Molecule & PEM Stack & Pros and Cons \\
\hline \multicolumn{4}{|l|}{ Confocal-Based Techniques } \\
\hline $\begin{array}{l}\text { FRAP (Fluorescence Recovery } \\
\text { after Photobleaching) }\end{array}$ & $\begin{array}{l}\text { Protein Lysozyme (Lys), } \\
\text { Human Serum Albumin (HSA) }\end{array}$ & $\begin{array}{l}\text { poly(L lysine) } \\
\text { /hyaluronic acid (PLL/HA) }\end{array}$ & $\begin{array}{l}\text {-Best for studying lateral } \\
\text { transport in } \mu \mathrm{m} \text { thick films } \\
\text { - Cannot be used for out of } \\
\text { plane molecular dynamics }\end{array}$ \\
\hline $\begin{array}{l}\text { FRET } \\
\text { (Forster Resonance Energy Transfer) }\end{array}$ & Chitosan $(\mathrm{CH})$ & $\begin{array}{l}\text { Chitosan/heparin } \\
\text { (CH/HEP) }\end{array}$ & $\begin{array}{l}\text {-Useful for biomolecule probing } \\
\text { with in I0-100 nm PEM stack } \\
\text { - Out of plane dynamics can be studied } \\
\text { - Require careful interpretation of data }\end{array}$ \\
\hline $\begin{array}{l}\text { FCS } \\
\text { (Fluorescence Correlation } \\
\text { Spectroscopy) }\end{array}$ & Rhodamine B (RB) & $\begin{array}{l}\text { poly(sodium } \\
\text { 4-styrenesulfonate)and poly } \\
\text { (diallydimethlyammonium } \\
\text { chloride) ) } \\
\text { (PSS/PDDA) }\end{array}$ & Reveals Fast molecular dynamics \\
\hline \multicolumn{4}{|l|}{ Recent Innovative Techniques } \\
\hline $\begin{array}{l}\text { Depth-profile X-ray } \\
\text { photoelectron spectroscopy (XPS) }\end{array}$ & Chitosan $(\mathrm{CHI})$ & $\begin{array}{l}\text { poly(ethylene oxide) } \\
\text { /poly(acrylic acid (PEO/PAA) }\end{array}$ & $\begin{array}{l}\text {-Can determine interlayer diffusion } \\
\text { within } 15 \mathrm{~nm} \text { thick films } \\
\text {-Require specialized source e.g., } \\
\text { C } 60+\text { cluster ions for minimal damage }\end{array}$ \\
\hline $\begin{array}{l}\text { Modified SERS Surface-enhanced } \\
\text { Raman spectroscopy }\end{array}$ & Methylene Blue ( MB) & $\begin{array}{l}\text { poly(allylamine } \\
\text { hydrochloride)poly(acrylic } \\
\text { acid (PAH/PAA) }\end{array}$ & $\begin{array}{l}\text {-Minimize diffusion and long } \\
\text { term storage of Raman active probes } \\
\text {-Require careful Preparation }\end{array}$ \\
\hline VERTICAL Diffusion & $\begin{array}{l}\text { Bovine Serum Albumin (BSA) \& } \\
\text { Biotin }\end{array}$ & $\begin{array}{l}\text { poly(allylamine hydrochloride) } \\
\text { poly(acrylic acid (PAH/PAA) }\end{array}$ & $\begin{array}{l}\text { Probe vertical transport in } \\
\text { PEM with thickness in the range } \\
\text { of } 100-200 \mathrm{~nm} \text {. }\end{array}$ \\
\hline
\end{tabular}

To investigate out-of-plane transport in thin polyelectrolyte multilayers, other optical techniques such as the Fluorescence Resonance Energy Transfer (FRET) and Fluorescence Correlation Spectroscopy (FCS) are better alternatives. ${ }^{12-16}$ For example, out-ofplane transport of chitosan $(\mathrm{CH})$ has been observed in films of $\mathrm{CH}$ and heparin (HEP) by using FRET. ${ }^{12}$ Here, $\mathrm{CH}$ molecules were labelled by either a donor dye or an acceptor dye. Donor-acceptor energy transfer is extremely sensitive to the distance between the fluorophores and therefore serves as a reporter of changes in the proximity of labelled polymers as they diffuse. These FRET experiments indicated that $\mathrm{CH}$ molecules diffused within the film with a transport coefficient that was not significantly sensitive to the deposition $\mathrm{pH}$ and solution ionic strength. Thus by utilizing FRET, polymer dynamics can be monitored in the vertical direction with significantly improved spatial resolution compared to FRAP.

Fluorescence Correlation Spectroscopy is also among the optical technique, which provides valuable information on the diffusive polymeric exchange in solution surrounding PEM stack. ${ }^{14-16}$ FCS is also known as Single Molecule Confocal Microscopy as it is very sensitive to very small molecular interactions within the PEM. The lateral transport of Rhodamine $\mathrm{B}(\mathrm{RB})$ was studied in the PEM films of sodium poly(sodium 4-styrenesulfonate)(PSS) and poly (diallydimethlyammonium chloride) (PDDA) by FCS. ${ }^{16} \mathrm{FCS}$ is able to provide information about molecular transport across the PEM stack at different temporal scales of several sub-microseconds to several hundred milliseconds. All the confocal-based techniques require the fluorescent modification of molecules being tracked and give indirect measurements of transport characteristics of PEMs.

\section{Recent innovations in probing bio-molecular transport \\ Depth-profile XPS}

Techniques such as X-ray photoelectron spectroscopy (XPS) with depth-profile option have proven to be capable of probing transport in inorganic materials. ${ }^{17}$ However, the use of such techniques for studying transport in soft polymeric thin films suffers from several challenges. XPS can damage either the polymer layers or change the structural organization of bioactive species under investigation. Therefore, in order to generate error-proof data, specialized conditions are required for using XPS for probing bio-molecular transport. For example, $\mathrm{C} 60+$ cluster ions have recently been used for generating high resolution depth-profiling XPS analysis to minimize the damage to soft materials. Here, efficacy of hydrogen bonding is compared with electrostatic interactions for blocking interlayer transport of Chitosan (CHI) across multilayer stack of poly (ethylene oxide)/ poly (acrylic acid (PEO/PAA). By using this high resolution depthprofile XPS, it has been verified that $(\mathrm{CHI})$ can diffuse easily into the hydrogen-bonded region i.e. (PEO/PAA), where as a single layer of poly(allylamine hydrochloride) $\mathrm{PAH}$, which is an electrostatic blocking-layer, is sufficient in stopping the CHI diffusion into the PEM stack. Such a design of PEM stack, with interlayer blocking capability is proposed to be highly beneficial for sustained drug release applications.

\section{Modified SERS}

Recently, molecular transport has been studied by utilizing specially designed Surface-enhanced Raman spectroscopy (SERS) substrates..$^{18}$ Here, the SERS substrates have been modified with polyelectrolyte multilayers of poly (allylamine hydrochloride) (PAH) and poly (acrylic acid (PAA) by layer-by-layer (LbL) process. Wrapping these SERS probes with PEM has proven to be efficient for their long term storage by reducing the transport of reporter molecules into the surrounding environment. To study this effect, methylene blue was used as an optical reporter molecule, which was encapsulated with the PEM stack. SERS signals were recorded from these Raman active reporters over a period of 5 weeks. To minimize transport and improve the long-term storage stability of these nanoprobes, thiol coating and cross-linking strategies were used. In both cases, 
molecular transport is significantly diminished. Such a method of polyelectrolyte wrapping on SERS substrates is an easy way to protect them from harsh biological environment for long observation period.

\section{Vertical -diffusion measurement}

This is one of the most recent optical techniques for extracting bio-molecular transport across PEM in transverse direction (normal to the substrate). This technique offers the ability to study transport in ultrathin polymer films (thickness $100-200 \mathrm{~nm}$ ). ${ }^{19}$ This technique is based on the distance-dependent periodic modulation of fluorescence intensity emitted from the fluorescently labelled molecule. Thus, diffusion profile can be extracted by measuring the fluorescence emission from the different polymer thickness. This technique has been employed to study the transport of bovine serum albumin (BSA) and biotin across the polymeric stack of poly (allylamine hydrochloride) $\mathrm{PAH} /$ poly (acrylic acid) PAA. The experimental demonstrations were in agreement with a proposed mathematical model which was validated by probing molecular transport in the PEM under different environmental conditions to either enhance or suppress the molecular diffusion across polymer films. This technique also provides a clear picture of molecular loading into the PEM.

\section{Conclusion}

As PEM serves as the most effective stimuli-sensitive reservoir platform for various bio-medical applications, so it is essential to gain insights into the loading and releasing of biomolecules across the multilayer stack. Most of the research in this field is mainly focussed on understanding the physico-chemical aspects of PEM. This minireview provide a good overview of current research for tracking the location and availability of bio-molecules across the PEM stack, which is essential for variety of applications ranging from biomedical applications to other surface coatings, designing effective membrane separations etc. Confocal-based techniques that involve the fluorescently tagged diffusing molecules and some recent innovations in this field have been discussed in this paper. This knowledge opens new facets of bio-molecular interactions within PEM. Such monitoring or tracking of molecules is essential for creating desired carrier architectures for drug delivery applications where on-time, ondemand availability of bio-molecules is crucial. Overall, this effort will greatly expand the knowledge about the dynamical aspects of biomolecular transport occurring in PEM stack and provides new possibilities for designing the novel class of carrier materials for better therapeutic applications.

\section{Acknowledgements}

We do not have any acknowledgment for the study.

\section{Conflicts of interest}

All authors have no conflicts of interest to declare.

\section{Funding}

None.

\section{References}

1. Keeney M, Jiang XY, Yamane M, et al. Nanocoating for biomolecule delivery using layer-by-layer self-assembly. $J$ Mater Chem $B$ 2015;3(45):8757-8770.
2. Su X, Kim BS, Kim SR, et al. Layer-by-Layer Assembled Multilayer Films for Transcutaneous Drug and Vaccine Delivery. ACS Nano. 2009;3(11):3719-3729.

3. Ganda A, Chacona HD, Tassel PRV, et al. Nanotemplated polyelectrolyte films as porous biomolecular delivery systems. Application to the growth factor BMP-2. Biomatter. 2014;4:e28823.

4. Jiang B, Barnett JB, Li B. Advances in polyelectrolyte multilayer nanofilms as tunable drug delivery systems. Nanotechnology Sci App. 2009;2:21-27.

5. Ibarz BG, Dahne L, Donath E, et al. Smart Micro- and Nanocontainers for Storage, Transport, and Release. Adv Mater. 2001;1(17):1324-1327.

6. Cao S, Zhang Y, Zhou L, et al. Stimuli-responsive controlled release and molecular transport from hierarchical hollow silica/ polyelectrolyte multilayer formulations. J Mater Chem B. 2014;2(41):7243-7249.

7. Axelford D, Koppel DE, Schlessinger J, et al. Mobility measurement by analysis of fluorescence photobleaching recovery kinetics. Biophys $J$. 1976;16:1055-1069.

8. Velka N, Uhlig K, Vikulinaa A, et al. Mobility of lysozyme in poly(1lysine)/hyaluronic acid multilayer films. Colloids and Surfaces B: Biointerfaces. 2016;147:343-350.

9. Nazaran P, Bosio V, Jaeger W, et al. Lateral Mobility of Polyelectrolyte Chains in Multilayers. J Phys Chem B. 2007;111(29): 8572-8581.

10. Vogt C, Ball V, Mutterer J, et al. Mobility of Proteins in Highly Hydrated Polyelectrolyte Multilayer Films. $J$ Phys Chem B. 2012;116(17):5269-5278.

11. Uhlig K, Madaboosi N, Schmidt S, et al. 3d localization and diffusion of proteins in polyelectrolyte multilayers. Soft Matter. 2012;8(47):11786-11789.

12. Lundin M, Blomberg E, Tilton RD. Polymer Dynamics in Layer-by-Layer Assemblies of Chitosan and Heparin. Langmuir. 2010;26(5):3242-3251.

13. Lee L, Johnston APR, Caruso F. Probing the Dynamic Nature of DNA Multilayer Films Using Forster Resonance Energy Transfer. Langmuir. 2012;28(34):12527-12535.

14. Casoli A, Schonhoff M. Fluorescence Correlation Spectroscopy as a Tool to Investigate Single Molecule Probe Dynamics in Thin Polymer Films. Biol Chem. 2001;382(3):363-369.

15. Bhattacharya S, Dey A, Chowdhury A. Probing Differential Hydration of Poly (vinylpyrrolidone) Thin Films Using Tracer Mobility: An Insight from Fluorescence Correlation Spectroscopy. J Phys Chem B. 2014;118(19):5240-5249.

16. Carr JK, Himes RD, Keung CH, et al. Heterogeneous Translational Dynamics of Rhodamine B in Polyelectrolyte Multilayer Thin Films Observed by Single Molecule Microscopy. Langmuir. 2009; 25(14):8330-8339.

17. Gilbert JB, Rubner MF, Cohen RE. Depth-profiling X-ray photoelectron spectroscopy (XPS) analysis of interlayer diffusion in polyelectrolyte multilayers. PNAS. 2013;110(17):6651-6656.

18. DeVetter BM, Sivapalan ST, Patel DD, et al. Observation of Molecular Diffusion in Polyelectrolyte-Wrapped SERS Nanoprobes. Langmuir. 2014;30:8931-8937.

19. Pahal S, Raichur AM, Varma MM. Subdiffraction-Resolution Optical Measurements of Molecular Transport in Thin Polymer Films. Langmuir. 2016;32(22):5460-5467. 